\title{
Universality of delocalization in unconventional dirty superconducting wires with broken spin-rotation symmetry
}

\author{
P. W. Brouwer \\ Laboratory of Atomic and Solid State Physics, Cornell University, Ithaca, New York 14853-2501 \\ A. Furusaki \\ Yukawa Institute for Theoretical Physics, Kyoto University, Kyoto 606-8502, Japan \\ C. Mudry \\ Paul Scherrer Institute, CH-5232 Villigen PSI, Switzerland \\ and Yukawa Institute for Theoretical Physics, Kyoto University, Kyoto 606-8502, Japan
}

(Received 21 February 2002; published 31 January 2003)

\begin{abstract}
In dirty superconducting wires, quasiparticle states at the Fermi level need not be exponentially localized if spin-rotation symmetry is broken [Brouwer et al., Phys. Rev. Lett. 85, 1064 (2000)]. Here we present evidence that not-localized states are generic in the thick-wire limit, while for wires of finite thickness delocalization requires fine tuning of the disorder, consistent with earlier results of Motrunich et al. [Phys. Rev. B 63, 224204 (2001)]. The thick-wire limit is defined as the simultaneous limit where the length $L$ of the wire and the number $N$ of propagating channels at the Fermi energy are both taken to infinity with their ratio held fixed.
\end{abstract}

DOI: 10.1103/PhysRevB.67.014530

PACS number(s): 74.25.Fy, 72.15.Rn, 73.20.Fz, 73.23.-b

Localization properties of weakly disordered normalmetal wires depend first and foremost on the fundamental symmetries of the wire: presence or absence of time-reversal symmetry and spin-rotation symmetry. In addition to these, two more symmetries can play an important role in the characterization of quantum wires: chiral symmetry and particlehole symmetry. The former is relevant for lattice models with randomness in the hopping amplitudes only, while the latter applies to superconducting wires or normal-metal wires in the proximity of a superconductor.

Is symmetry the only player determining the localization properties? For normal-metal wires, it is generally agreed on that this is the case when the disorder is weak. For superconducting wires, different views have been published in the literature. Advocates of field-theoretic, random-matrix, or Fokker-Planck approaches have mostly considered the symmetry classification as sufficient, thus arriving at a division of superconducting wires into four universality classes. ${ }^{1-7}$ These are labeled C, CI, D, and DIII, and are characterized by the presence or absence of time-reversal symmetry and spin-rotation symmetry. ${ }^{1}$ For the classes D and DIII, where spin-rotation symmetry is broken, it was found that wave functions are "critical" if disorder is weak, rather than exponentially localized, on length scales beyond a characteristic crossover scale $N l$, where $N$ is the number of propagating channels in the wire and $l$ is the mean free path. Here "critical" indicates algebraic decay of the average conductance, opposed to exponential decay if wave functions are localized. Disorder is "weak" if $k_{F} l \gg 1, k_{F}$ being the Fermi wave vector. On the other hand, using a numerical transfer matrix method to calculate localization lengths in single-channel wires and strong-disorder renormalization-group arguments, Motrunich et al. found that, if spin-rotation symmetry is broken, the localization length only diverges if the disorder is fine tuned. ${ }^{8}$ They concluded that symmetry alone is not sufficient to characterize disordered wires with particle-hole symmetry. Such a finding is consistent with the expectation that, in a single-channel wire, weak spin-orbit scattering or weak superconducting correlations cannot destroy the localized phase that exists in the presence of spin-rotation symmetry and without superconductivity.

In this paper we take a more detailed look at this apparent contradiction for the case of class D where, in addition to spin-rotation symmetry, time-reversal symmetry is broken as well. We consider both regimes $L \gg N l$ and $L \lesssim N l$. For thick wires $(N \gg 1)$, the regime $l \ll L \ll N l$ corresponds to the diffusive regime. For thin wires ( $N$ of order unity), one has $N l \sim l$, and there is no diffusive regime. Our main conclusion - to be supported by numerical and analytical arguments below-is that, as argued in Ref. 8, for quantum wires of finite thickness and broken spin-rotation symmetry, fine tuning of the disorder is required in order to obtain a diverging localization length. However, we shall also argue that in the limit $N \rightarrow \infty$ of "thick" quantum wires symmetry alone is sufficient to determine the localization properties. 9 We note that this criterion is not different from that in normal wires. ${ }^{10}$ Thus, as far as the classification based on symmetry is concerned, superconducting disordered wires are no less universal than ordinary normal-metal wires. In the final analysis, realization of the quasi-one-dimensional critical behavior of class D demands fine tuning of microscopic parameters for a wire of finite thickness but becomes generic in the thick-wire limit provided the disorder is sufficiently weak. We reach this conclusion by studying the competition between localization in the standard unitary class (no superconductivity) and criticality in class D that occurs upon increasing the strength of superconducting correlations in the wire. However, the same scenario applies to the crossover between the localized phase of class $\mathrm{C}$ and the critical phase of class $\mathrm{D}$ upon breaking of the spin-rotation symmetry.

As in Ref. 7, our starting point is the Hamiltonian 


$$
\mathcal{H}=\mathcal{K}+\mathcal{V}, \quad \mathcal{K}=\sigma_{0} \otimes \gamma_{0} \otimes \tau_{3} \otimes \mathbb{1}_{N} i v_{F} \partial_{x},
$$

where $\sigma_{0}$ is the $2 \times 2$ unit matrix in the spin grading, $\gamma_{0}$ is the $2 \times 2$ unit matrix in particle-hole grading, and $\tau_{3}$ is the Pauli matrix in left/right mover grading. The kinetic energy $\mathcal{K}$ describes the propagation of right and left moving quasiparticles in $N$ channels at the Fermi level. The "potential" $\mathcal{V}(x)$ is an $8 N \times 8 N$ matrix that accounts for the presence of both disorder and superconducting correlations. In particle/ hole $(\gamma)$ grading it reads

$$
\mathcal{V}=\left(\begin{array}{cc}
v & \Delta \\
-\Delta^{*} & -v^{\mathrm{T}}
\end{array}\right),
$$

where $v(\Delta)$ is a Hermitian (antisymmetric) $4 N \times 4 N$ matrix, representing the impurity potential (superconducting order parameter). The form (2) of the potential $\mathcal{V}$ ensures that the Hamiltonian $\mathcal{H}$ obeys particle-hole symmetry: $\mathcal{H}=$ $-\gamma_{1} \mathcal{H}^{\mathrm{T}} \gamma_{1}{ }^{1}$ In addition, $\mathcal{H}$ (and hence $\mathcal{V}$ ) may obey timereversal invariance $\mathcal{H}=\mathcal{T} \mathcal{H}^{*} \mathcal{T}^{-1}$, with $\mathcal{T}=i \tau_{1} \otimes \sigma_{2}$.

In Ref. 7 it was assumed that the potentials $\Delta$ and $v$ in Eq. (2) are Gaussian white-noise potentials with the same variance. It was for this choice of disorder that the "critical" conductance statistics was found. Motrunich et al. have argued that this choice is special: As soon as the variances of $\Delta$ and $v$ are no longer equal, the conductance will decay exponentially with length $L$. In addition, they showed that if a term proportional to $v_{F} \tau_{2} / 2 l_{s}$ is added to the Hamiltonian, which corresponds to a staggering of the hopping amplitude in a lattice version of the problem, ${ }^{11}$ the localization length is finite even if $v$ and $\Delta$ have the same variance.

Below we shall analyze the effects of a different variance of $\Delta$ and $v$ and of staggering on the localization length, and discuss how these effects change with the thickness of the wire. We will first address the diffusive regime, where an analytical treatment is possible, and then the localized regime, where we support our arguments with numerical simulations.

Different variances of $\Delta$ and $v$. For simplicity, we assume that time-reversal symmetry and spin-rotational symmetry are completely broken. Hence we take the potentials $v$ and $\Delta$ as Gaussian white-noise, with vanishing means and with variances

$$
\begin{gathered}
\left\langle v_{i j}(x) v_{k l}^{*}(y)\right\rangle=\frac{v_{F}^{2}}{8 N l_{v}} \delta_{i k} \delta_{j l} \delta(x-y), \\
\left\langle\Delta_{i j}(x) \Delta_{k l}^{*}(y)\right\rangle=\frac{v_{F}^{2}}{8 N l_{\Delta}}\left(\delta_{i k} \delta_{j l}-\delta_{i l} \delta_{j k}\right) \delta(x-y) .
\end{gathered}
$$

Here $l_{v}$ and $l_{\Delta}$ are the mean free paths for scattering from potential disorder and from (fluctuations of) the superconducting pair potential $\Delta$, respectively. When $l_{\Delta}=l_{v}$, the exact solution of Ref. 7 applies. We will refer to this special case as the pure class $\mathrm{D}$ and consider the generic case $l_{\Delta}$ $\neq l_{v}$ as a point in the crossover between the standard unitary ensemble and class D. (The standard unitary ensemble corresponds to the absence of superconducting correlations, $l_{\Delta}$ $=\infty$.) The combined mean free path $l$ for scattering from either $v$ or $\Delta$ is $l^{-1}=l_{v}^{-1}+l_{\Delta}^{-1}$. In the special case $l_{\Delta}=l_{v}$, i.e., in the pure class $\mathrm{D}$, the ensemble average dimensionless conductance $\langle g\rangle$ in the diffusive regime $L \ll N l$ reads $^{7}$

$$
\langle g\rangle=\frac{4 N l}{L}+\frac{1}{3}+\mathcal{O}\left(\frac{L}{N l}\right) .
$$

For $L \gg N l$, the average conductance decays algebraically, whereas the "typical conductance" $\exp (\langle\ln g\rangle)$ decreases proportional to $\exp \left[-(2 L / \pi N l)^{1 / 2}\right],{ }^{7}$

$$
\langle g\rangle=\sqrt{\frac{8 N l}{\pi L}},\langle\ln g\rangle=-\sqrt{\frac{2 L}{\pi N l}} .
$$

For the standard unitary ensemble $l_{\Delta}=\infty$ one has $\langle g\rangle$ $=4 N l / L+\mathcal{O}(L / N l)$ in the diffusive regime, while in the localized regime $\langle g\rangle \propto e^{-L / 8 N l},\langle\ln g\rangle=-L / 2 N l+\mathcal{O}(1) .{ }^{12}$

Let us now consider the generic case $l_{v} \neq l_{\Delta}<\infty$, i.e., a point in the crossover between the standard unitary ensemble and the pure class D. At a fixed but large $l_{\Delta} \gg l_{v}$, this same crossover can also take place as a function of the wire length $L$. In that case, short $L$ corresponds to the standard unitary ensemble, while class D statistics might be found for large $L$. To find the relevant length scale for that crossover we calculate the average conductance in the diffusive regime. Following the method of Ref. 13 we derive scaling equations for the averages of certain traces of transmission and reflection matrices $t$ and $r$ of the wire. For $l_{\Delta}, L \gg l$ and up to corrections of relative order $(L / N l)$ the relevant scaling equations read

$$
\begin{aligned}
& 4 N l \partial_{L}\left\langle\operatorname{tr} t^{\dagger} t\right\rangle=-\left\langle\operatorname{tr} t^{\dagger} t\right\rangle^{2}+2[\left.\left\langle\operatorname{tr}\left(r^{\dagger} r t^{\dagger} t-\gamma_{3} r^{\dagger} \gamma_{3} r t^{\dagger} t\right)\right\rangle\right], \\
& 4 N l \partial_{L}\left\langle\operatorname{tr}\left(1-\gamma_{3} r^{\dagger} \gamma_{3} r\right)\right\rangle=-\left\langle\operatorname{tr}\left(1-\gamma_{3} r^{\dagger} \gamma_{3} r\right)\right\rangle^{2}+\left(\xi_{\Delta} / l\right)^{2}, \\
& 4 N l \partial_{L}\left\langle\operatorname{tr} \gamma_{3} r^{\dagger} \gamma_{3} r t^{\dagger} t\right\rangle=-2\left\langle\operatorname{tr}\left(1-\gamma_{3} r^{\dagger} \gamma_{3} r\right)\right\rangle \\
& \times\left\langle\operatorname{tr} \gamma_{3} r^{\dagger} \gamma_{3} r t^{\dagger} t\right\rangle, \\
& 4 N l \partial_{L}\left\langle\operatorname{tr} r^{\dagger} r t^{\dagger} t\right\rangle=- 2\left\langle\operatorname{tr} t^{\dagger} t\right\rangle\left\langle\operatorname{tr} r^{\dagger} r t^{\dagger} t\right\rangle,
\end{aligned}
$$

where we abbreviated $\xi_{\Delta}=\sqrt{l l_{\Delta} / 8}$ and $\gamma_{3}$ is the Pauli matrix in particle-hole grading. Solving Eqs. (6) we find the average conductance $\langle g\rangle=\left\langle\operatorname{tr} t^{\dagger} t\right\rangle$ for $l_{\Delta}, L \gg l,{ }^{14}$

$$
\langle g\rangle=\frac{4 N l}{L}+\frac{1}{3}+\frac{\xi_{\Delta}^{2}}{L^{2}}-\frac{\xi_{\Delta}}{L} \operatorname{coth} \frac{L}{\xi_{\Delta}}+\mathcal{O}\left(\frac{L}{N l}\right) .
$$

The length scale $\xi_{\Delta}$ marks the crossover between the standard unitary class and class D: For $L \ll \xi_{\Delta}$ we recover the result $\langle g\rangle=4 N l / L+\mathcal{O}(L / N l)$ of the standard unitary ensemble, while for $L \gg \xi_{\Delta}$ Eq. (7) simplifies to the class D result (4). As Eq. (7) is only valid in the diffusive regime $L \ll N l$, one can only expect to observe the class D result (4) if $\xi_{\Delta}$ is smaller than $N l$. In the thick-wire limit this poses no real constraint, and we conclude that, in the thick-wire limit, any finite mean free path $l_{\Delta}$ will eventually give rise to a weak localization correction to the conductance according to class D if the wire is sufficiently long.

To find what happens in the localized regime we now compare the crossover length scale $\xi_{\Delta}$ derived above and the localization length $4 \mathrm{Nl}$ in the standard unitary class. If $\xi_{\Delta}$ 
$\gg N l$, the wave functions are still governed by the unitary class on the scale $N l$, not by class $\mathrm{D}$, when localization sets in. Hence there is no room for class D physics to play a role and the localization length is that of the standard unitary class. On the other hand, if $\xi_{\Delta} \ll N l$, the statistics of wave functions and conductance is given by that of class D at scales $\xi_{\Delta}$ and beyond. Therefore if $\xi_{\Delta} \ll N l$ we expect that the wave function and conductance statistics for $L \gg N l$ is critical, as in class D. If $N l$ and $\xi_{\Delta}$ are comparable, the conductance statistics in the localized regime is best characterized as in the "crossover" between the two universality classes: The localization length is expected to be larger than $4 N l$, but still finite.

In order to make these statements more precise, we look at the normalized inverse localization length,

$$
\chi(\sigma)=-\frac{\langle\ln g\rangle}{\sigma}, \quad \sigma=\frac{L}{2 N l} .
$$

In the standard unitary ensemble, one has $\chi \rightarrow 1$ as $L \rightarrow \infty$ with $N, l_{\Delta}$, and $l_{v}$ held fixed; critical conductance statistics implies $\chi(\infty)=0$. A finite value $0<\chi(\infty)<1$ corresponds to exponentially localized wave functions, but with a localization length that is larger than in the standard unitary ensemble. Taking the limit $L \rightarrow \infty$ at fixed values of $N, l_{\Delta}$, and $l_{v}$ corresponds to a point somewhere in the crossover between the standard unitary ensemble and class D if $l_{\Delta} \neq l_{v}$. As such a choice of parameters corresponds to a finite distance from the pure class $\mathrm{D}$, we expect $\chi(\infty)>0$ in this case, corresponding to exponentially localized wave functions. This agrees with the claim of Motrunich et al., ${ }^{8}$ who found critical statistics at the point $l_{\Delta}=l_{v}$ only.

In the thick-wire limit, however, the picture is different. The thick-wire limit is defined as the simultaneous limit $L$ $\rightarrow \infty$ and $N \rightarrow \infty$, keeping $\sigma=L / 2 N l$ fixed. The limit $N \rightarrow \infty$ arises as a semiclassical limit, when the Fermi wavelength is much smaller than the diameter of the wire, or by increasing wire diameter. In the latter case, the diameter should not exceed the transverse localization length. Based on our calculation for the diffusive regime, we surmise that in the thick-wire limit the crossover between the standard unitary class and class $\mathrm{D}$ is described by a two-parameter scaling function $\chi^{*}(\sigma, \alpha)$, which is obtained from $\chi$ by taking the limit $L, N \rightarrow \infty$, at fixed values of the scaling variables $\sigma$ and $\alpha=2 N l / \xi_{\Delta} \cdot{ }^{15}$ The second scaling variable $\alpha$ is the ratio of the localization length $4 \mathrm{Nl}$ in the standard unitary class and twice the diffusive crossover length scale $\xi_{\Delta}$ (the proportionality constant is arbitrary as long as it is of order 1). As a function of $\alpha, \chi^{*}$ interpolates between the standard unitary ensemble for $\alpha=0$ and the critical conductance statistics of class D if $\alpha \rightarrow \infty$. Note that taking the thick-wire limit at any finite and fixed ratio of $\xi_{\Delta}$ and $l$ (or, alternatively, of $l_{\Delta}$ and l) corresponds to the case $\alpha=\infty$, i.e., automatically realizes class D. Thus, in the sense of the scaling limit defined above, the standard unitary fixed point is "unstable," whereas the class D fixed point is "attractive." It is only by allowing $\xi_{\Delta} / l$ to scale at least as fast as $N$ that one can probe nonclass-D physics in the thick-wire limit.

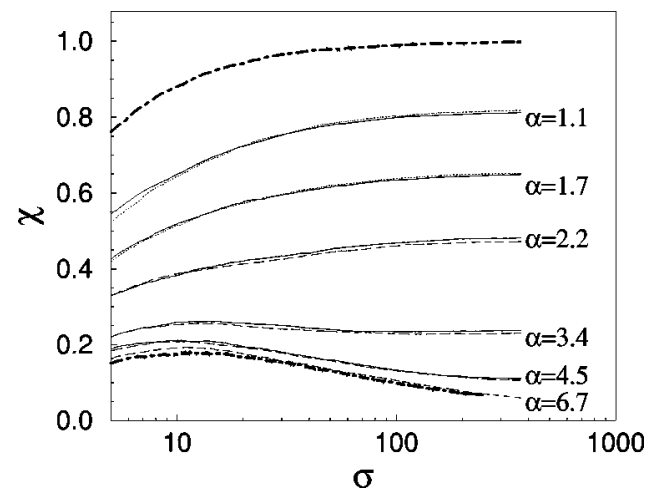

FIG. 1. Normalized inverse localization length $\chi=-\langle\ln g\rangle / \sigma$ versus $\sigma=L / 2 N l$. Solid curves are for $l_{\Delta}=201 l\left(\xi_{\Delta}=5.01 l\right)$ and $N=4,6$, from top to bottom. Dotted and dashed curves are for $l_{\Delta}$ $=101 l\left(\xi_{\Delta}=3.6 l\right)$ with $N=2,3,4,6,8$, and $l_{\Delta}=26 l\left(\xi_{\Delta}=1.8 l\right)$ with $N=2,3,4,6$, respectively. Thick dash-dot curves at the top and bottom are for the standard unitary class $\left(l_{\Delta}^{-1}=0\right)$ and $N=1$ and for the pure class $\mathrm{D}\left(l_{\Delta}=2 l\right)$ and $N=3$, respectively. Curves with equal values of the scaling variable $\alpha=2 N l / \xi_{\Delta}$ but different $\xi_{\Delta} / l$ or $N$ almost coincide; the remaining small differences between them are attributed to finite- $N$ effects. Corresponding values of $\alpha$ are listed to the right of each set of curves with the same $\alpha$.

We verified the above scenario with numerical simulations of $\chi$ as a function of $\sigma$ for a discretized version of the model (1). The discretized model is obtained from Eq. (1) by the replacement of the continuous potential $\mathcal{V}(x)$ by the sum $\Sigma_{j} \mathcal{V}_{j} \delta(x-a j)$, where $a$ is a microscopic length scale. The delta functions $\delta(x-y)$ in Eq. (3) are replaced by Kronecker deltas $a^{-1} \delta_{j j^{\prime}}$. In Fig. 1, results are shown for different values of $l_{\Delta} / l$ and for different $N$. We took an average over $>2000$ realizations of the disorder potential. Our conclusions are confirmed by the numerical data: First, curves for the same value of $\alpha=2 N l / \xi_{\Delta}=4 N\left(2 l / l_{\Delta}\right)^{1 / 2}$ approach a well-defined limit for large $N$, confirming that $\alpha=2 \mathrm{Nl} / \xi_{\Delta}$ is indeed the true crossover parameter. Second, in the crossover between the two classes, one still observes localized behavior, but with a significantly enhanced localization length, which increases as $\alpha$ is increased.

Staggering. Staggering is described by the addition of a term $v_{F} \tau_{2} / 2 l_{s}$ to the Hamiltonian (1). Without the potential term $\mathcal{V}$ in Eq. (1), the effect of staggering would be to create a gap of size $v_{F} / l_{s}$ around the Fermi energy $\varepsilon=0$. The effect of a random potential $\mathcal{V}$ is to create states inside this gap. Below we address the localization properties of such states at the Fermi level $\varepsilon=0$ if the random potential $\mathcal{V}$ is of the form (2) with nonzero superconducting correlations $\Delta$ and without spin-rotational invariance and time-reveral symmetry. Based on a combination of analytical and numerical arguments, we argue that eigenstates at the Fermi level remain critical (as in the absence of staggering), provided that the staggering is sufficiently weak [see Eq. (10) below] and the thick-wire limit is taken.

To study the effect of staggering on the $\varepsilon=0$ eigenstates of the Hamiltonian (1), we first investigate the conductance in the diffusive regime. Following the method of Ref. 13, we construct scaling equations for the moments $R_{n, m}$ $=\left\langle\operatorname{tr} r^{n} r^{\dagger m}\right\rangle$. To leading order in $N$, they read 


$$
\begin{aligned}
\partial_{L} R_{n, m}= & \frac{n}{l_{s}}\left(R_{n+1, m}-R_{n-1, m}\right)+\frac{m}{l_{s}}\left(R_{n, m+1}-R_{n, m-1}\right) \\
& -\frac{2(n+m)}{l} R_{n, m} \\
& +\frac{1}{4 N l} \sum_{p=0}^{n-1} \sum_{q=0}^{m-1}\left[R_{p, q}\left(R_{n-1-p, m-1-q}+R_{n-p, m-q}\right)\right. \\
& \left.+R_{n-p, m-q}\left(R_{p+1, q+1}+R_{p, q}\right)\right] \\
& -\frac{1}{N l} \sum_{p=1}^{n-1}(n-p) R_{p, 0} R_{n-p, m} \\
& -\frac{1}{N l} \sum_{q=1}^{m-1}(m-q) R_{0, q} R_{n, m-q} .
\end{aligned}
$$

Although the scaling equations (9) do not form a closed set, a solution can be constructed as a series expansion in $l / l_{s}$. For sufficiently weak staggering,

$$
l_{s}>l
$$

and for lengths $L \gg l$, the perturbation series in $l / l_{s}$ truncates after the second order, and one finds the simple solution

$$
\langle g\rangle=\left\langle\operatorname{tr}\left(1-R_{1,1}\right)\right\rangle=\frac{2}{\sigma}+\mathcal{O}(1),
$$

where $\sigma$ is now defined as

$$
\sigma=\frac{L}{2 N l\left[1-\left(l / l_{s}\right)^{2}\right]} .
$$

Hence, in the thick-wire limit, staggering causes a renormalization of the scaling length $\sigma$, but does not remove the existence of a diffusive regime. If staggering exceeds the critical strength (10), there is no diffusive regime in which $\langle g\rangle$ is inversely proportional to wire length at $\varepsilon=0$. The existence of a critical staggering strength is consistent with numerical simulations of periodic-on-average single-channel quantum wires by Deych et al. ${ }^{16}$

Based on this result for the diffusive regime, we propose that in the simultaneous limit $N \rightarrow \infty, L \rightarrow \infty$ the normalized inverse localization length $\chi=-\langle\ln g\rangle / \sigma$ approaches the same scaling limit $\chi^{*}(\sigma)$ as in the absence of staggering, if $\sigma$ is given by Eq. (12). In Fig. 2 we show the result of numerical simulations for the discretized version of Eq. (1) with staggering. We have compared the cases $l_{v} / l_{\Delta}=1$ or 0 , with or without (weak) staggering and find that for sufficiently large $N$ all curves $\chi$ versus $\sigma$ with the same symmetry of the Hamiltonian collapse. That is, curves with $l_{v} / l_{\Delta}$ $=1$ (or, in fact, any finite ratio $l_{v} / l_{\Delta}$ ) approach the class D result, while curves with $l_{v} / l_{\Delta}=0$ approach the result of the standard unitary class. Small $N$ curves are slightly different, which again indicates the absence of universality when a diffusive regime is absent.

In conclusion, our analytical and numerical results confirm the claim of Motrunich et al. that the conductance sta-

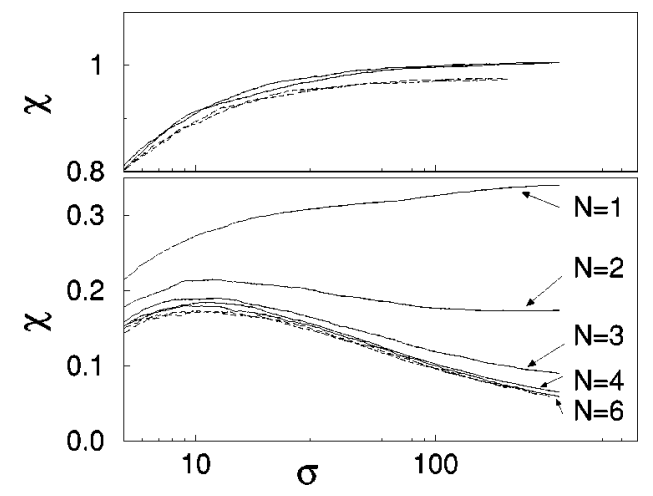

FIG. 2. Normalized inverse localization length $\chi=-\langle\ln g\rangle / \sigma$ versus scaled wire length $\sigma$ with and without staggering. With staggering, $\sigma$ is given by Eq. (12), without staggering $\sigma=L / 2 N l$. Upper panel shows results for normal quantum wires $\left(l_{\Delta}=\infty\right)$. Solid curves are for $l_{v}=l=0.80 l_{s}$ and for $N=4,6$; dashed curves are without staggering and $N=4,6$. The slight difference (less than $5 \%$ ) between curves with and without staggering is attributed to a small deviation from Eq. (12) due to the finite disorder strength in the simulations. As all curves for different $N$ collapse, no single curves are labeled. Lower panel shows results for superconducting quantum wires with $l_{\Delta}=l_{v}$. Solid curves are for $l_{v}=l_{\Delta}=2 l=1.60 l_{s}$ and $N=1,2,3,4$, and 6. Dashed curves are without staggering and $N=3, N=4$. They almost coincide with the curve with staggering and $N=6$ and are not labeled individually.

tistics in the localized regime is not determined by symmetry alone for a superconducting quantum wire with a small number $N$ of propagating channels at the Fermi level. ${ }^{8}$ Such universality is only achieved for "thick" quantum wires with $N \gg 1$. Which situation is relevant depends on the physical system at hand. For quasiparticle states at the edges of superconducting planes in high- $T_{c}$ superconductors, the nonuniversal scenario of small $N$ may be more applicable, while we expect that the universal scenario of large $N$ is more appropriate for dirty unconventional superconducting wires or normal-metal wires in the proximity of a superconductor. Possible relevant perturbations to the critical behavior in the thick-wire limit of the superconducting class $\mathrm{D}$ are disorder sufficiently strong to induce transverse localization, restoration of the spin-rotation symmetry, or breaking of the particle-hole symmetry (e.g., by tuning the chemical potential away from the the Fermi level or by electron-electron interactions). All of these perturbations are expected to restore the localized phase at large length scales.

We would like to point out that the universality — or lack thereof-for the localization properties in superconducting quantum wires is not different from the case of normal-metal wires. In both cases, universality is expected in the thickwire limit only. Or, in the language of scaling flow: In both cases, the fixed point with less symmetry is attractive, but the flow towards the fixed point is only complete if the thickwire limit is taken. In that respect, note that for the crossover between the standard unitary class and the superconducting class D considered in this paper, the superconducting class is the class of less symmetry. That fact constitutes a qualitative 
difference between class D and the three chiral classes, which also may exhibit critical conductance statistics for wire length $L \gg N l$. ${ }^{11}$ In contrast to the superconducting class $\mathrm{D}$, the chiral classes have very high symmetry, which makes them rather unstable and which will render observation of the critical conductance statistics in these classes more difficult.

We would like to thank K. Damle and I. A. Gruzberg for discussions. This work was supported in part by the NSF under Grant No. DMR 0086509, by the Sloan and Packard foundations (P.W.B.), and by a Grant-in-Aid for Scientific Research on Priority Areas (A) from the Ministry of Education, Culture, Sports, Science and Technology (No. 12046238) (A.F.). P.W.B. and A.F. thank the Institute for Theoretical Physics in Santa Barbara for its hospitality during the final stages of this work. C.M. thanks the Yukawa Institute for Theoretical Physics in Kyoto for its hospitality during the final stages of this work.
${ }^{1}$ A. Altland and M. R. Zirnbauer, Phys. Rev. Lett. 76, 3420 (1996); Phys. Rev. B 55, 1142 (1997).

${ }^{2}$ A. Altland, B. D. Simons, and D. Taras-Semchuk, Pis'ma Zh. Eksp. Teor. Fiz. 67, 21 (1998) [JETP Lett. 67, 22 (1998)].

${ }^{3}$ R. Bundschuh, C. Casanello, D. Serban, and M. R. Zirnbauer, Nucl. Phys. B 532, 689 (1998); Phys. Rev. B 59, 4382 (1999).

${ }^{4}$ T. Senthil, M. P. A. Fisher, L. Balents, and C. Nayak, Phys. Rev. Lett. 81, 4704 (1998); T. Senthil and M. P. A. Fisher, Phys. Rev. B 60, 6893 (1999).

${ }^{5}$ T. Senthil and M. P. A. Fisher, Phys. Rev. B 61, 9690 (2000); N. Read and D. Green, ibid. 61, 10267 (2000).

${ }^{6}$ M. Bocquet, D. Serban, and M. R. Zirnbauer, Nucl. Phys. B 578, 628 (2000).

${ }^{7}$ P. W. Brouwer, A. Furusaki, I. A. Gruzberg, and C. Mudry, Phys. Rev. Lett. 85, 1064 (2000).

${ }^{8}$ O. Motrunich, K. Damle, and D. A. Huse, Phys. Rev. B 63, 224 204 (2001).

${ }^{9}$ Interestingly, the thick-wire limit is a necessary step in the derivation of the one-dimensional nonlinear sigma model, see, e.g., A. D. Mirlin, A. Müller-Groeling, and M. R. Zirnbauer, Ann. Phys. (N.Y.) 236, 325 (1994). The Fokker-Planck approach is often used without the thick-wire limit, though equivalence of the Fokker-Planck and field-theoretic approach is only estab- lished if the thick-wire limit is taken; see P. W. Brouwer and K. M. Frahm, Phys. Rev. B 53, 1490 (1996).

${ }^{10}$ To illustrate this point, note that an infinitesimal magnetic field already changes the symmetry of the Hamiltonian from orthogonal to unitary. However, for a wire of finite thickness, one needs a finite magnetic field to reach the unitary class, while in the thick-wire limit an infinitesimal field is sufficient. See, e.g., H. Schomerus and C. W. J. Beenakker, Phys. Rev. Lett. 84, 3927 (2000).

${ }^{11}$ P. W. Brouwer, C. Mudry, B. D. Simons, and A. Altland, Phys. Rev. Lett. 81, 862 (1998).

${ }^{12}$ C. W. J. Beenakker, Rev. Mod. Phys. 69, 731 (1997).

${ }^{13}$ C. Mudry, P. W. Brouwer, and A. Furusaki, Phys. Rev. B 62, 8249 (2000); 63, 129901(E) (2001).

${ }^{14}$ For $l_{\Delta} \approx l_{v}$ or $l_{\Delta} \ll l_{v}$ the class $\mathrm{D}$ result (4) is obtained already for wire lengths $L$ of the order of the mean free path.

${ }^{15}$ Note that the limit $\sigma \rightarrow \infty$ of the scaling function $\chi^{*}(\sigma, \alpha)$ is a different quantity than the limit $N \rightarrow \infty$ of the Lyapunov exponent, which is defined by taking the limit $L \rightarrow \infty$ before the limit $N \rightarrow \infty$ in Eq. (8).

${ }^{16}$ L. I. Deych, D. Zaslavsky, and A. A. Lisyansky, Phys. Rev. Lett. 81, 5390 (1998). 